\title{
Prevention and therapy of acute and chronic wounds using NPWT devices during the COVID-19 pandemic, recommendation from The NPWT Working Group
}

Tomasz Banasiewicz, Rolf Becker, Adam Bobkiewicz, Marco Fraccalvierri, Wojciech Francuzik, Martin Hutan, Mike Laukoetter, Marcin Malka, Bartosz Mańkowski, Zsolt Szentkereszty, Csaba Toth, Lenka Veverkova, Sudheer Karlakki, John Murphy, Maciej Zieliński

\section{ORIGINAL ARTICLES}

\begin{abstract}
Recent SARS-CoV-2 pandemic leading to a rapidly increasing number of hospitalizations enforced reevaluation of wound management strategies.

The optimal treatment strategy for patients with chronic wounds and those recovering from emergency and urgent oncological surgery should aim to minimize the number of hospital admissions, as well as the number of surgical procedures and decrease the length of stay to disburden the hospital staff and to minimize viral infection risk.

One of the potential solutions that could help to achieve these goals may be the extensive and early use of NPWT devices in the prevention of wound healing complications.

Single-use NPWT devices are helpful in outpatient wound treatment and SSI prevention (ciNPWT) allowing to minimize in-person visits to the health care center while still providing the best possible wound-care. Stationary NPWT should be used in deep SSI and perioperative wound healing disorders as soon as possible. Patient's education and telemedical support with visual wound healing monitoring and video conversations have the potential to minimize the number of unnecessary in-person visits
\end{abstract}

Manuscript received 11.04.2020; revised 16.04.2020. This work did not receive any financial support.

Author affiliations: Department of General, Endocrinological Surgery and Gastrointestinal Oncology, Poznan University of Medical Sciences, Poznan, Poland, (TB, AB); Departmentleiter Revisionsendoprothetik, Eduardus Krankenhaus Köln, Germany , (RB); Plastic Surgery Unit, Aso Citta' della Saliute Della Scienza Di Torino, Italy, (MF); Department of Dermatology, Venereology and Allergology, Charité - Universitätsmedizin Berlin, corporate member of Freie Universität Berlin, Humboldt-Universität zu Berlin, and Berlin Institute of Health, (WF); Surgical department, LK Hainburg, Hainburg a.d. Donau, Austria, (MH); Department of General and Visceral Surgery, Mathias-Spital Rheine, Frankenburgstrasse 31, 48431 Rheine, Germany, (ML); PODOS Wound Care Clinic, Warsaw, Poland, (MM); Div. of Trauma, Burns and Plastic Surgery, Poznan University of Medical Sciences, (BM); Faculty of Medicine, Institute. of Surgery, Debrecen, University of Debrecen, Hungary, (ZS); Medical and Health Science Center, Institute of Surgery, Dept. of Vascular Surgery, University of Debrecen, Hungary, (CT); Masaryk University Brno Ist Surgical Dpt. St Anne's University Hospital Brno (FNUSA-ICRC), Czech Republic, (LV); Consultant Orthopaedic and Arthoplasty SurgeRJAH Orthopaedic Hospital NHS FT, Oswestry, United Kingdom, Honorary Senior Lecturer (Keele University), (SK); Nightingale Breast Centre, Manchester University Hospitals Foundation NHS Trusts, Manchester, United Kingdom, (JM); Dept of Vascular Surgery Poznan University of Medical Science Poland, (MZ)

*Correspondence to: Tomasz Banasiewicz: tbnanasiewicz@op.pl in patients with wounds and therefore substantially increase the level of care.

Keywords-NPWT, Covid-19, SARS-CoV-2, chronic wounds

\section{INTRODUCTION}

$\mathbf{T}$ HE epidemiological situation in the world caused by the SARS-CoV-2 virus leads to a rapidly increasing number of hospitalizations. Hospital wards are being converted into dedicated COVID-19 wards what brings many sudden changes in the system and treatment strategy. There are many recommended procedures for the prevention of surgical site infections (SSI), but only a few deals with the treatment of complications, and especially patients in home care. The current general strategy is to minimize the number of nonessential hospitalizations for three main reasons:

1) providing intensive care units the capacity for COVID19 patients requiring intensive care;

2) preserving medical staff due to the shortage of medical personnel

3) reducing the risk of infection for hospitalized patients and medical staff

SSI symptoms after abdominal and chest injury surgery may camouflage asymptomatic SARS-CoV-2 infection especially in the era of COVID-19 pandemic. 1 Therefore minimizing hospitalization time may decrease the risk of viral transmission in post-surgical patients.

\section{GeNerAL STRATEGY}

The optimal treatment strategy for patients with chronic wounds and those recovering from emergency and urgent oncological surgery should aim to minimize the number of hospital admissions because of the surgical reasons described in detail in the ERAS protocol, $\stackrel{2]}{2}$ as well as the number of surgical procedures. On the other hand, the hospitalization time should be optimized to be as short as possible to 

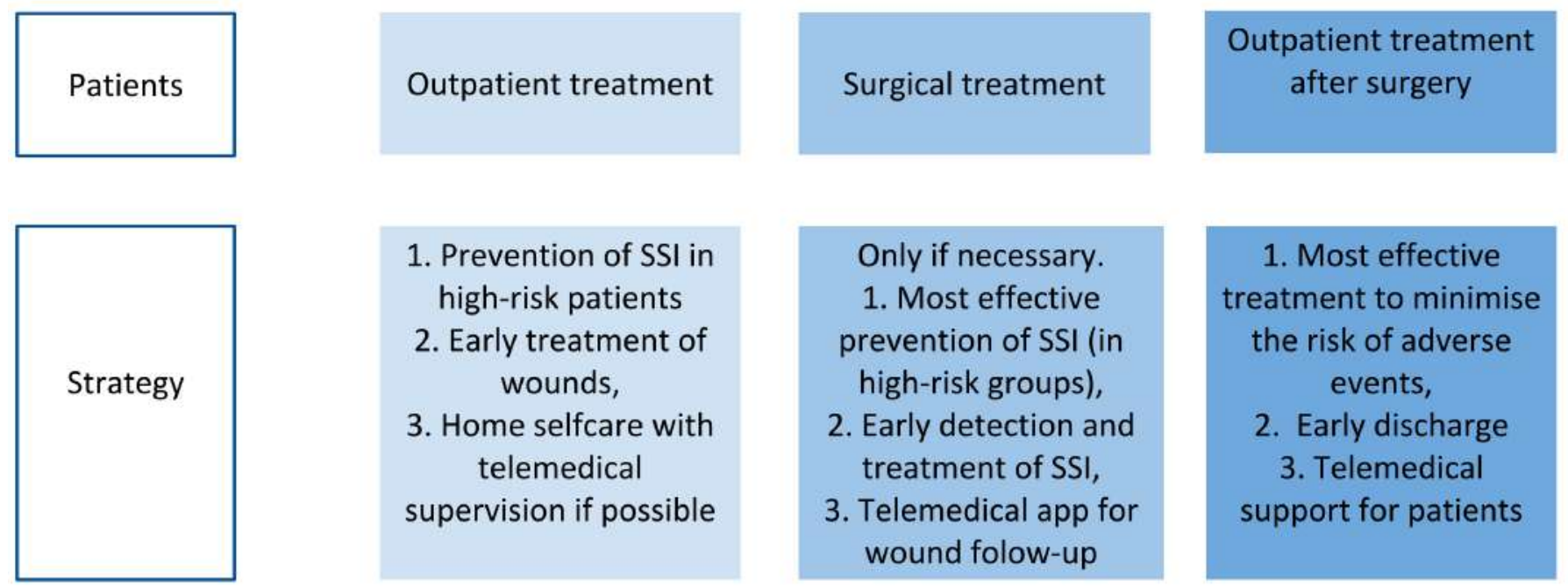
1. Prevention of SSI in high-risk patients
2. Early treatment of wounds,
3. Home selfcare with telemedical supervision if possible
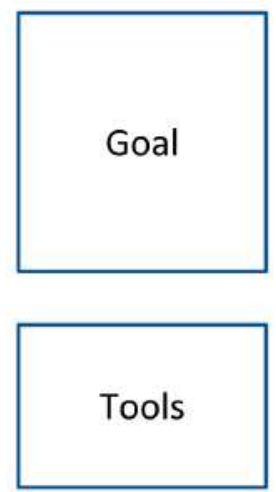

Support wound
healing, reduce
numbers of
complications, reduce
risk of hospitalization
Single use NPWT, stationary NPWT in selected cases
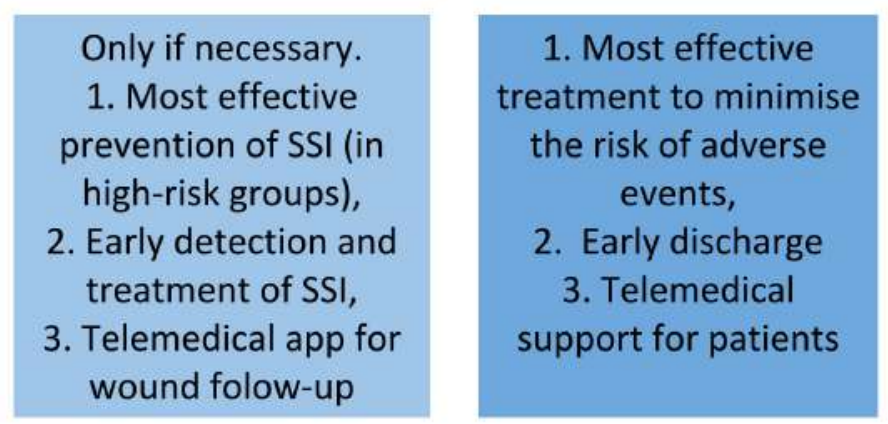
To lower the risk of SSI, avoid reoperations, and prolonged hospitalization, early discharge

\section{Single use NPWT - ciNPWT, stationary NPWT including INPWT}

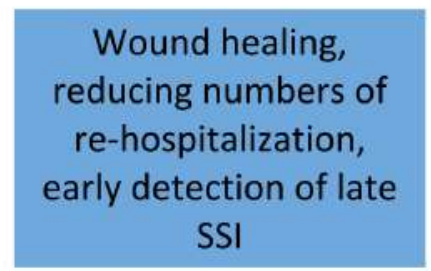

Single use NPWT, stationary NPWT in selected cases

Figure 1. The treatment strategy in three most common settings (columns) during the SARS-CoV-2 pandemic.

disburden the hospital staff and to minimize viral infection risk. One of the potential solutions that could help to achieve these goals may be the extensive and early use of Negative Pressure Wound Therapy (NPWT) devices in the prevention of wound healing complications ${ }^{3}$ (mainly surgical site infections - $\mathrm{SSH}^{4}$ or wound dehiscence), particularly among higher-risk patients,,$\sqrt[5]{]}$ those with complex incisions with ort without prosthetic devices underlying the closed incision. ${ }^{6} \mathrm{Li}$ et al. proved that patients undergoing NPWT after open abdominal surgery had SSI less frequently 9 and the formation of enteroatmospheric fistulae were also less frequent in patients receiving. 10

The relative liberal use of ciNPWT, may well be advantageous for patients who are discharged as an inpatient earlier than normal to free up capacity for acute COVID beds may continue to receive good quality healthcare at home.11

This strategy may be highly important in a group of patients undergoing urgent surgery or those with wounds classified as contaminated. Based on recent studies, ciNPWT significantly reduced the incidence rate of SSI in colorectal, inflammatory bowel disease patients or groin vascular surgery, as well as other disciplines such as Orthopaedic and Plastic Surgery 12 NPWT could also be used more extensively in tearly wound healing complications and wound infections allowing a rapid, safe and effective inpatient discharge. Prevention of SSI using ciNPWT (closed incision NPWT) can lead to reduce the incidence of SSI, and also the number of wound dressing changes ${ }^{[15}$ (disburdening medical staff, and minimizing contact with the patients). In septic and complicated wounds, iNPWT (instillation NPWT) should be more commonly applied. It was stated that the utility of NPWT with instillation in complicated and non-healed wounds was associated with a significant decrease in bacterial overload reduction, time to wound closure and hospital discharge $\frac{16}{16}$ The principles of the treatment strategy are shown on (Fig. 1).

NPWT can be used as soon as possible in wound healing disturbances, especially those caused by infections. The stationary device initially applied in hospitalized patients can be continued in an ambulatory setting using a singleuse portable NPWT device. Single-use ciNPWT devices are widely available on the market, intuitive, and easy to use. The education of patients on how to remove a vacuum wound dressing, in selected well-collaborating patients, can be very 


\begin{tabular}{|l|c|}
\hline $\begin{array}{l}\text { Out-patients treatment (telemedicine!) } \\
\text { Main goal: avoid of chronic wounds, avoid } \\
\text { complications, avoid of hospitalizations, } \\
\text { reduce time of treatment. }\end{array}$ & $\begin{array}{c}\text { Surgery (only if necessary) } \\
\text { Main goal: avoid of complications, avoid of } \\
\text { reoperation, reduce the hospitalization } \\
\text { time, avoid of chronic wound }\end{array}$ \\
\hline
\end{tabular}

Prevention of wounds in high risk groups (diabetic foot, pressure ulcers, venous ulcers)

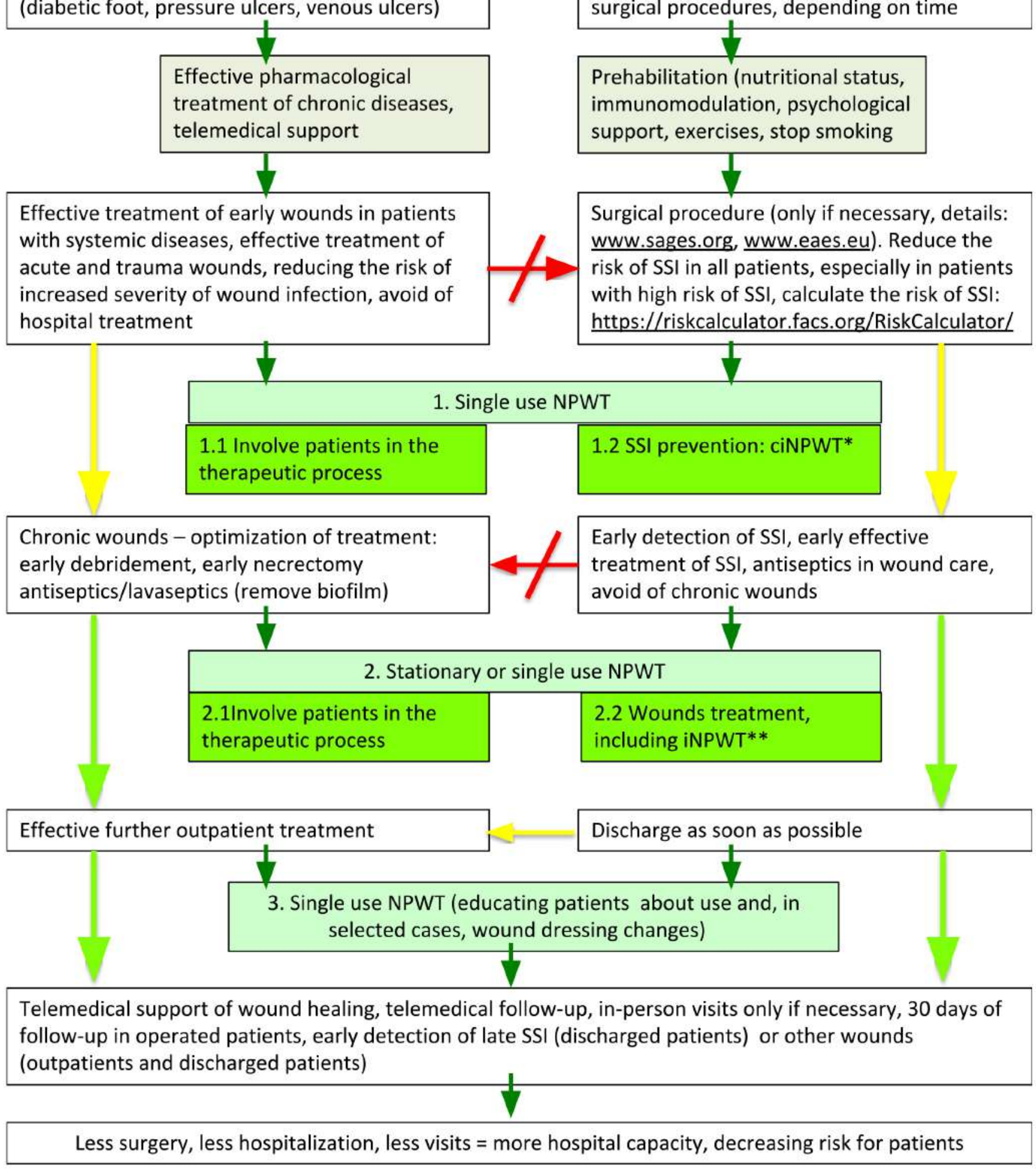

Figure 2. Proposed patient flow in a surgery hospital department. 
helpful and is essential when dealing with the dressing's seal leak during NPWT at home. Current evidence support the home use of single-use NPWT (sNPWT) due to high patientsatisfaction. 17

Dowset et al. provided data on the clinical and economic benefit of sNPWT in patients with chronic wounds allowing to free up medical staff! ${ }^{18}$ Similar benefits have been shown

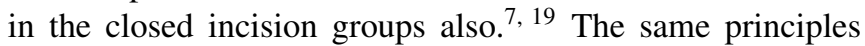
will apply to acute and surgical wounds during the COVID19 pandemic. This is especially important as there is evidence that the effectiveness of sNPWT is similar in inpatient and outpatient setting. 20

The telemedical support for patients should lead to a good postoperative wound control and can be used in an outpatient treatment manner. Options with the possibility of digital photography transfer are preferable. The optimal flow of patients (out- and inpatients) is shown on (Fig. 2). The main problems associated with the wound healing process are listed below.

\section{Problem: Management of outpatients with wounds}

Goal: In patients with acute or chronic wounds the ambulatory treatment should be as effective as inpatient therapy while reducing the number of in-person visits. Solution (using NPWT):

1) The prophylactic ${ }^{21}$ use of single-use NPWT devices if possible (reducing the number of wound dressings changes, making treatment more effective) is advised.

2) In chronic venous leg ulcers NPWT should be combined with compression therapy (using either bandaging or compression stockings. 22

3) Patients should be educated on minimizing the risk of SARS-CoV-2 infection.

4) Telemedical suppor ${ }^{23}$ for patients during wound dressing self-removal, dressing leaks, and, in selected cases, in wound dressing changes is essential, with secure video conversations as the gold standard.

\section{Problem: Management of surgical patients (emergency and urgent oncologic procedures) and SSI}

Goals:

1) reduction of the SSI severity and frequency;

2) effective treatment of SSI;

3) avoidance of reoperations;

4) early discharge

Solution (using NPWT):

1) NPWT in all patients with complicated wounds, (e.g. open abdomen, 24 open fractures ${ }^{25}$ ), and ciNPWT in highrisk patients for SSI, complex wounds and those associated with a prosthesis are recommended (if available and possible also in other patients); In order to minimize the costs of the ciNPWT - alternative low-cost methods can be advised.26

2), 3) NPWT therapy should be introduced as soon as possible in SSI (according to CDC classification: ${ }^{27}$ superficial incisional SSI - single-use NPWT, stationary NPWT; deep incisional SSI — stationary NPWT (consider instillation -
iNPWT); organ or space SSI — stationary NPWT (consider instillation — iNPWT)

4) optimal wound healing should be provided to patients with a higher risk of developing SSI (obesity or cachexia, ASA 3 and 4, immunosupression, steroids, cigarette smoking, comorbidities) by using an effective wound dressing suitable for discharge - single-use NPWT combined with patient education on wound dressing self-removal and, in selected cases, in wound dressing changes. Telemedical support for theses patients is essential to minimize in-person visits.23 Patient should be educated that in case of skin infection presenting with pain, heat, redness, swelling or purulent discharge at the incision site, they should seek immediate consultation via telemedical support.

Even if lower risk for SSI is calculated, ciNPWT should be considered as first line treatment option for wound management. Such management may reduce the risk for developing SSI to the minimum and prevent readmission or complications during outpatient care.

In cases where patients would not be able to follow telemedical guidance on wound dressing changes or in more complicated dressing requiring in-person visits, it is highly advisable to prolong the intervals between dressing changes in order to minimize the number of in-person visits. NPWT is highly suitable to prolong dressing change intervals 17 and can be recommended to achieve this.

\section{Problem: Follow-up surveillance of outpatients after surgery}

Goal: To facilitate wound follow-up with detection of SSI after discharge, reduce the number of in-person visits, and provide the most effective wound healing support. Solution (using NPWT):

1) The use of single pocket NPWT devices if possible (reducing the number of wound dressings changes, making(e) treatment more effective) is advised.

2) Patients should be educated on minimizing the risk of SARS-CoV-2 infection education.,

3) Telemedical support for patients during wound dressing self-removal and, in selected cases, in wound dressing changes with secure video conversations is essential.

\section{Conclusion}

In order to reduce the risk of viral transmission, early treatment of wound healing complications, and reducing the risk of SSI using NPWT is advisable, especially during the SARS-CoV-2 pandemic. NPWT should be considered in wound healing disorders and their prevention as the therapy can effectively decrease the number of complications, reduce the number of surgical interventions, decrease the length of stay, reduce the number of wound dressings changes, reduce the number of contacts between patients and medical staff and disburden the already decompressed healthcare system.

Single-use NPWT devices are helpful in outpatient wound treatment and SSI prevention (ciNPWT) allowing to minimize in-person visits to the health care center while still providing the best possible wound-care. Stationary NPWT 
should be used in deep SSI and perioperative wound healing disorders as soon as possible. Patient's education and telemedical support with visual wound healing monitoring and video conversations have the potential to minimize the number of unnecessary in-person visits in patients with wounds and therefore substantially increase the level of care.

\section{REFERENCES}

[1] M. Khazaei, R. Asgari, E. Zarei, Y. Moharramzad, H. Haghighatkhah, and M. S. Taheri, "Incidentally Diagnosed COVID-19 Infection in Trauma Patients; a Clinical Experience," Archives of Academic Emergency Medicine, vol. 8, no. 1, p. 31, 2020

[2] M. Melnyk, R. G. Casey, P. Black, and A. J. Koupparis, "Enhanced recovery after surgery (ERAS) protocols: Time to change practice?" Canadian Urological Association Journal, pp. 342-348, Oct. 2011, publisher: Canadian Urological Association Journal. [Online]. Available: https://doi.org/10.5489/cuaj.11002

[3] F. Sexton, D. Healy, S. Keelan, M. Alazzawi, and P. Naughton, "A systematic review and meta-analysis comparing the effectiveness of negative-pressure wound therapy to standard therapy in the prevention of complications after vascular surgery," International Journal of Surgery, vol. 76, pp. 94-100, Apr. 2020, publisher: Elsevier BV. [Online]. Available: https://doi.org/10.1016/j.ijsu.2020.02.037

[4] H.-Z. Li, X.-H. Xu, D.-W. Wang, Y.-M. Lin, N. Lin, and H.-D. $\mathrm{Lu}$, "Negative pressure wound therapy for surgical site infections: a systematic review and meta-analysis of randomized controlled trials," Clinical Microbiology and Infection, vol. 25, no. 11, pp. 1328-1338, Nov. 2019, publisher: Elsevier BV. [Online]. Available: https://doi.org/10.1016/j.cmi.2019.06.005

[5] V. Strugala and R. Martin, "Meta-Analysis of Comparative Trials Evaluating a Prophylactic Single-Use Negative Pressure Wound Therapy System for the Prevention of Surgical Site Complications," Surgical Infections, vol. 18, no. 7, pp. 810-819, Oct. 2017, publisher: Mary Ann Liebert Inc. [Online]. Available: https: //doi.org/10.1089/sur.2017.156

[6] R. Holt and J. Murphy, "Pico ${ }^{\mathrm{TM}}$ incision closure in oncoplastic breast surgery: a case series," British Journal of Hospital Medicine, vol. 76, no. 4, pp. 217-223, 2015.

[7] G. W. Irwin, G. Boundouki, B. Fakim, R. Johnson, L. Highton, D. Myers, R. Searle, and J. A. Murphy, "Negative pressure wound therapy reduces wound breakdown and implant loss in prepectoral breast reconstruction," Plastic and Reconstructive Surgery-Global Open, vol. 8, no. 2, p. e2667, 2020.

[8] S. Karlakki, M. Brem, S. Giannini, V. Khanduja, J. Stannard, and R. Martin, "Negative pressure wound therapy for management of the surgical incision in orthopaedic surgery: a review of evidence and mechanisms for an emerging indication," Bone $\mathcal{E}$ joint research, vol. 2, no. 12, pp. 276-284, 2013.

[9] P.-Y. Li, D. Yang, D. Liu, S.-J. Sun, and L.-Y. Zhang, "Reducing Surgical Site Infection with Negative-Pressure Wound Therapy After Open Abdominal Surgery: A Prospective Randomized Controlled Study," Scandinavian Journal of Surgery, vol. 106, no. 3, pp. 189195, Sep. 2016, publisher: SAGE Publications. [Online]. Available: https://doi.org/10.1177/1457496916668681

[10] K. Szmyt, \. Krokowicz, A. Bobkiewicz, B. Cybu|lka, W. Ledwosiński, M. Gordon, A. Alammari, T. Banasiewicz, and M. Drews, "Comparison of the Effectiveness of the Treatment Using Standard Methods and Negative Pressure Wound Therapy (NPWT) in Patients Treated with Open Abdomen Technique," Polish Journal of Surgery, vol. 87, no. 1, Jan. 2015, publisher: Index Copernicus. [Online]. Available: https://doi.org/10.1515/pjs-2015-0013

[11] S. Karlakki, A. Hamad, C. Whittall, N. Graham, R. Banerjee, and J. Kuiper, "Incisional negative pressure wound therapy dressings (inpwtd) in routine primary hip and knee arthroplasties: a randomised controlled trial," Bone E joint research, vol. 5, no. 8, pp. 328-337, 2016.

[12] T. Matatov, K. N. Reddy, L. D. Doucet, C. X. Zhao, and W. W. Zhang, "Experience with a new negative pressure incision management system in prevention of groin wound infection in vascular surgery patients," Journal of Vascular Surgery, vol. 57, no. 3, pp. 791-795, Mar. 2013, publisher: Elsevier BV. [Online]. Available: https://doi.org/10.1016/j.jvs.2012.09.037
[13] G. Pellino, G. Sciaudone, G. Candilio, F. Campitiello, F. Selvaggi, and S. Canonico, "Effects of a New Pocket Device for Negative Pressure Wound Therapy on Surgical Wounds of Patients Affected With Crohn's Disease," Surgical Innovation, vol. 21, no. 2, pp. 204212, Jul. 2013, publisher: SAGE Publications. [Online]. Available: https://doi.org/10.1177/1553350613496906

[14] A. U. Blackham, J. P. Farrah, T. P. McCoy, B. S. Schmidt, and P. Shen, "Prevention of surgical site infections in highrisk patients with laparotomy incisions using negative-pressure therapy," The American Journal of Surgery, vol. 205, no. 6, pp. 647-654, Jun. 2013, publisher: Elsevier BV. [Online]. Available: https://doi.org/10.1016/j.amjsurg.2012.06.007

[15] R. Kirsner, C. Dove, A. Reyzelman, D. Vayser, and H. Jaimes, "A prospective, randomized, controlled clinical trial on the efficacy of a single-use negative pressure wound therapy system, compared to traditional negative pressure wound therapy in the treatment of chronic ulcers of the lower extremities," Wound Repair and Regeneration, vol. 27, no. 5, pp. 519-529, 2019, eprint https://onlinelibrary.wiley.com/doi/pdf/10.1111/wrr.12727. [Online] Available: https://onlinelibrary.wiley.com/doi/abs/10.1111/wrr.12727

[16] A. Gabriel, J. Shores, C. Heinrich, W. Baqai, S. Kalina, N. Sogioka, and S. Gupta, "Negative pressure wound therapy with instillation: a pilot study describing a new method for treating infected wounds," International Wound Journal, vol. 5, no. 3, pp. 399413, Jun. 2008, publisher: Wiley. [Online]. Available: https: //doi.org/10.1111/j.1742-481x.2007.00423.x

[17] T. Hurd, P. Trueman, and A. Rossington, "Use of a portable, singleuse negative pressure wound therapy device in home care patients with low to moderately exuding wounds: a case series," Ostomy/Wound Management, vol. 60, no. 3, pp. 30-36, Mar. 2014

[18] C. Dowsett, J. Hampton, D. Myers, and T. Styche, "Use of PICO to improve clinical and economic outcomes in hard-to-heal wounds," Wounds Int, vol. 8, no. 2, pp. 52-58, 2017.

[19] L. M. Nherera, P. Trueman, and S. L. Karlakki, "Cost-effectiveness analysis of single-use negative pressure wound therapy dressings (snpwt) to reduce surgical site complications (ssc) in routine primary hip and knee replacements," Wound Repair and Regeneration, vol. 25, no. 3, pp. 474-482, 2017.

[20] J. Stryja, R. Staffa, D. Říha, K. Stryjová, and K. Nicielniková, "[Cost-effectiveness of negative pressure wound therapy in outpatient setting]," Rozhledy V Chirurgii: Mesicnik Ceskoslovenske Chirurgicke Spolecnosti, vol. 94, no. 8, pp. 322-328, Aug. 2015.

[21] C. Heard, W. Chaboyer, V. Anderson, B. M. Gillespie, and J. A. Whitty, "Cost-effectiveness analysis alongside a pilot study of prophylactic negative pressure wound therapy," Journal of Tissue Viability, vol. 26, no. 1, pp. 79-84, Feb. 2017, publisher: Elsevier BV. [Online]. Available: https://doi.org/10.1016/j.jtv.2016.06.001

[22] E. Wang, R. Tang, N. Walsh, L. Stopher, C. Bharat, S. Ponosh, and S. Jansen, "Topical negative pressure therapy and compression in the management of venous leg ulcers: A pilot study," Wound Practice $\mathcal{E}$ Research: Journal of the Australian Wound Management Association, vol. 25, no. 1, p. 36, Mar. 2017, publisher: Cambridge Publishing. [Online]. Available: https://search.informit.org/ documentSummary; $\mathrm{dn}=745540889743034 ;$ res=IELHEA

[23] A. Lumpkins and T. Stanton, "Benefits of a Patient-centered Remote Therapy Monitoring Program Focusing on Increased Adherence to Wound Therapy." Wounds: a compendium of clinical research and practice, vol. 31, no. 8, pp. E49-E53, 2019.

[24] A. L. Fowler and M. K. Barry, "Closed incision negative pressure therapy: Results of recent trials and recommendations for clinical practice," The Surgeon, Dec. 2019, publisher: Elsevier BV. [Online] Available: https://doi.org/10.1016/j.surge.2019.10.007

[25] M. C. Grant-Freemantle, J. Ryan, S. O. Flynn, D. P. Moloney, M. A. Kelly, E. I. Coveney, B. J. O'Daly, and J. F. Quinlan, "The Effectiveness of Negative Pressure Wound Therapy Versus Conventional Dressing in the Treatment of Open Fractures," Journal of Orthopaedic Trauma, p. 1, Feb. 2020, publisher: Ovid Technologies (Wolters Kluwer Health). [Online]. Available: https://doi.org/10.1097/bot.0000000000001750

[26] D. A. Walczak, M. Grajek, M. Zeman, T. Pałka, M. Kalkum, M. Dobrut, P. Drozdowski, R. Ulczok, K. Donocik, A. Maciejewski, and Krakowczyk, "Novel, self-made and cost-ective technique for closed-incision negative pressure wound therapy," Negative Pressure Wound Therapy Journal, vol. 7, no. 1, pp. 8-10, Mar. 2020. [Online]. Available: https://npwtj.com/index.php/npwtj/article/view/56

[27] T. C. Horan, R. P. Gaynes, W. J. Martone, W. R. Jarvis, and T. G. Emori, "CDC Definitions of Nosocomial Surgical Site 
Infections, 1992: A Modification of CDC Definitions of Surgical Wound Infections," Infection Control $\mathcal{E}$ Hospital Epidemiology, vol. 13, no. 10, pp. 606-608, Oct. 1992, publisher: Cambridge
University Press (CUP). [Online]. Available: https://doi.org/10.1017/ s0195941700015241 